\title{
Schwannome bénin intramandibulaire. \\ Rapport d'un cas et revue de la littérature
}

\section{Mandibular Schwannoma: a case report and review of the literature}

\author{
SAÏD KIMAKHE*, YVES HIRIGOYEN*, BERNARD GIUMELLI**.
}

\section{RÉSUMÉ}

Le schwannome bénin est une tumeur périphérique rare. Elle dérive des cellules de la gaine de Schwann. Cette tumeur à progression lente, affecte le plus souvent la région de la tête et du cou et survient généralement lors de la troisième ou la quatrième décade de la vie. II est rapporté le cas d'une lésion ostéolytique mandibulaire évoquant un vaste kyste périapical résiduel qui s'est avérée être à l'examen anatomopathologie un schwannome mandibulaire du nerf alvéolaire inférieur avec ses cellules caractéristiques de type Antoni A et de type Antoni B. La localisation intra-buccale des schwannomes est de faible incidence. Ces rares localisations, quand elles sont intraosseuses buccales, affectent le plus souvent la mandibule que le maxillaire. La revue de la littérature sur les schwannommes bénins ne rapporte que 40 cas seulement à localisation intramandibulaire (Med Buccale Chir Buccale 2002; 8: 37-44).

médecine buccale chirurgie buccale VOL. $8, \mathrm{~N}^{\circ}$ 2002 page 37

mots clés : Schwannome, Neurilemmome, Tumeur neurogène, Mandibule.

\section{SUMMARY}

Schwannoma or neurilemoma is a rare benign tumor derived from proliferation of Schwann cells of the neurilemma that surronds peripheral nerves. Most cases occur during the third and fourth decade of the life. The head and the neck are most commonly involved including soft tissues of the oral cavity. Bony lesions, which produce a radiolucent pattern, are rare and the mandible is more affected than maxilla. In this paper, the case of a 41 year old woman affected by an intramandibular schwannoma is reported and a review of the literature is prensented (Med Buccale Chir Buccale 2002; 8: 37-44).

key words : Schwannoma, Neurilemmoma, Mandible.

* Département de Chirurgie et de Médecine Buccales, UFR d'Odontologie de Nantes.

* Service d'Odontologie Restauratrice et d'Odontologie.

Demande de tirés à part :

Saïd Kimakhe Département de Chirurgie et de Médecine Buccales UFR d'Odontologie 44000 Nantes

Article reçu le 10 septembre 2000. Accepté pour publication le 25 octobre 2001. 
médecine buccale chirurgie buccale

)L. $8, \mathrm{~N}^{\circ} 1$

2001

page 38
Le schwannome ou neurilemmome est une tumeur neurogène périphérique rare. Elle dérive des cellules de Schwann dont l'origine est neuro-ectodermique. Le schwannome dit cellulaire, diffère du schwannome classique par son niveau cellulaire plus élevé, par le polymorphysme nucléaire et par la haute fréquence de son activité mitotique. C'est une tumeur bénigne, qui ne connaît pas en principe de transformation maligne. Un seul cas de dégénérescence maligne a été rapporté [1]. Cependant, plusieurs cas de schwannomes cellulaires ont été confondus avec certains schwannomes dit malins sans métastase [2].

Les schwannomes ont été regroupés dans une seule et même entité avec les neurofibromes, ils étaient classifiés comme des «faux neuromes», des neurofibromes ou encore des «neuronomes». Stout en 1935 [3] a proposé le terme de neurilemmome et, a classé les tumeurs neurogènes en dehors de toute considération histologique en neurofibrome (lésion isolée), neurofibromatose (lésion multiple, syndrome ou maladie de Von Recklinghausen), neurome (d'origine traumatique et d'amputation) et en neurilemmome ou neurinome.

D'autres classifications basées sur des données histo-pathologiques et immuno-histo-chimiques ont permis de différencier les schwannomes des neurofibromes [4]. Des études ultrastructurales réalisées par Sugimura et coll. [5] ont confirmé l'existence d'une lame basale, rendant de fait l'origine fibroblastique mésenchymateuse périneurale difficilement acceptable [6]. J unqueira et coll. [7] ont différencié ces tumeurs neurogènes en fonction de la nature du collagène qu'elles renferment, le neurilemmome (possédant du collagène de type III) et neurofibrome (possédant du collagène de type I et III).

La classification reconnue et adoptée actuellement a été rapportée par Reychler [8] :

1) Schwannome bénin ou neurilemmome, tumeur bénigne dérivant des cellules de Schwann d'origine neuro-ectodermique.

2) Schwannome ou neurilemmome ancien, variante du schwannome bénin, tumeur caractérisée par des remaniements dégénératifs (calcification, kystisation...).
3) Neurofibrome solitaire, tumeur qui se développe à partir des éléments conjonctifs de la gaine de Schwann et se rencontre rarement de manière isolée.

4) Neurofibromatose, comporte deux sous classes:

4.1) Neurofibromatose de type I, correspond au syndrome décrit par Von Recklinghasen. Elle se manifeste par des pigmentations mélaniques appelées «tâches de café au lait» au sein d'organes d'origine ectodermique (peau, système nerveux) ; elle s'accompagne d'atteintes ophtalmiques (hamartome irique) et de déficiences squelettiques (cypho-scoliose sévère à grand rayon). C'est une maladie hériditaire (probablement structurelle du chromosome 17), elle évolue sur le mode autosomique dominant à pénétrance variable (une naissance sur 2000 à 3300).

4.2) Neurofibromatose de type II, peu commune, elle présente des lésions cutanées plus rares et se caractérise par le développement de tumeurs bilatérales du nerf acoustique. C'est une maladie héréditaire qui évolue sur le mode autosomique dominant (une naissance sur 50000), liée probablement à une déficience de structure au niveau du chromosome 22.

5) Névromes ou neurome vrais (traumatique ; cutané encapsulé et palissadé ; muqueux multiple).

6) Myxome de la gaine nerveuse ou neurocthome.

\section{OBSERVATION}

Une patiente âgée de 41 ans est adressée pour des extractions des dents de sagesse supérieures (18 et 28) et pour un curetage d'une lésion ostéolytique intra-mandibulaire gauche qui se traduit par une image radioclaire appendue aux apex de la 36 extraite depuis peu sous anesthésie locorégionale (Fig. 1 ).

L'examen clinique montre une tuméfaction vestibulaire en regard de la 36 , sans fistule, indolore à la palpation, de consistance osseuse recouverte d'une muqueuse saine. Cette tuméfaction limitée ne s'accompagne pas de signe neurologique 
(signe de Vincent). L'image radioclaire est circonscrite et globalement homogène, elle paraît bilobée et bien délimitée par une paroi condensante nette et continue. Le canal mandibulaire qui est élargi en fuseau dans la région du l'angle est non visible au contact de la lésion. Les caractéristiques cliniques et radiologiques évoquent un vaste kyste périapical résiduel.

Le traitement proposé dans un premier temps est l'énucléation de la lésion par voie alvéolaire élargie et dans un deuxième temps les extractions des 18 et 28 sous anesthésie loco-régionale. La pièce opératoire après son énucléation est fixée dans du formol à $10 \%$ et adressée pour l'examen ana- tomopathologique. Cet examen de la pièce opératoire montre des zones de prolifération cellulaire de densité modérée parsemées de tissu à prédominance lymphocytaire au sein d'une coque fibrohyaline (Fig. 2). Ces zones cellulaires sont faites de cellules fusiformes en trousseaux, parfois orientées, à petits noyaux : les cellules d'Antoni $\mathrm{A}$ (a) ; et parfois désordonnées en fonction du plan de coupe (b) (Fig. 2). L'étude immuno-histo-chimique révèle une forte positivité à la protéine $\mathrm{S}$ 100 traduisant une différenciation nerveuse (Fig. 3). Cet aspect histologique évoque donc une tumeur nerveuse, un schwannome.

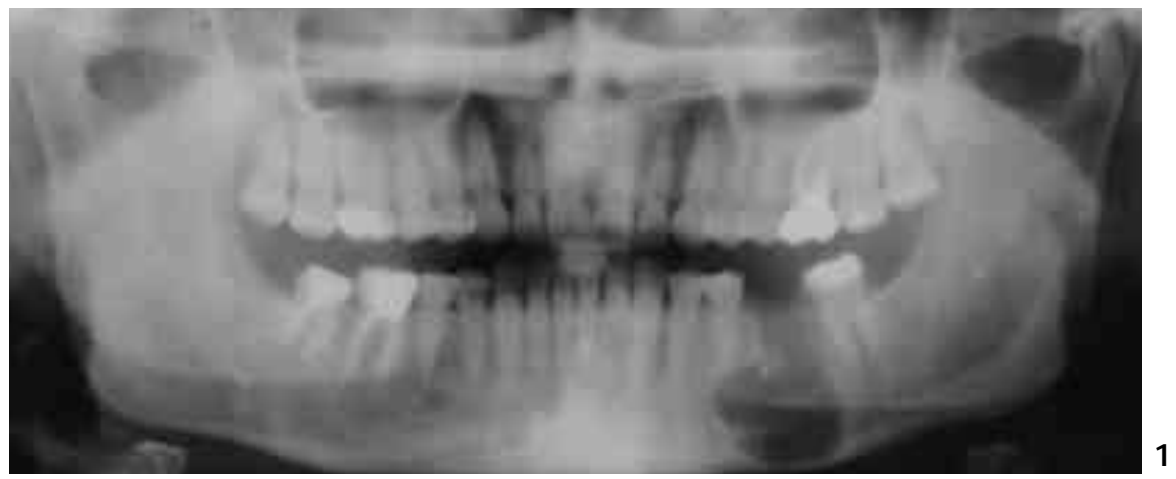

médecine buccale chirurgie buccale VOL. $8, \mathrm{~N}^{\circ}$ 2002 page 39
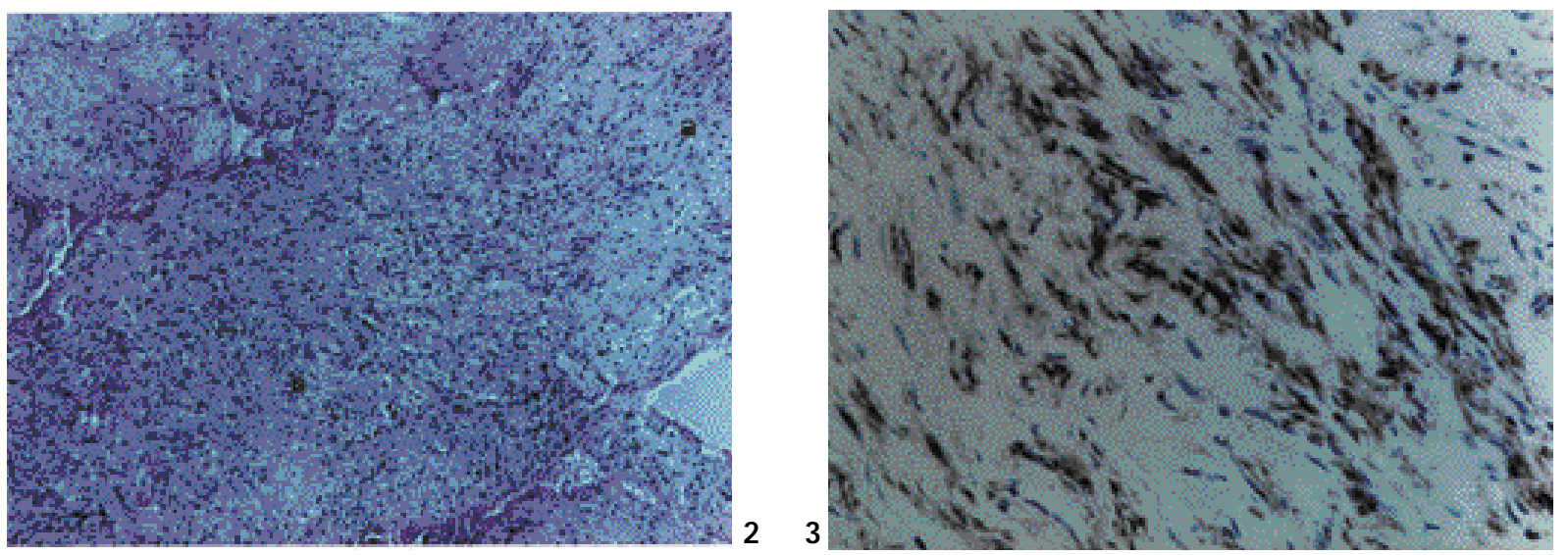

Figure 1 : Radiographie panoramique après extraction de la 36, montrant une lésion ostéolytique au niveau de la branche horizontale gauche et un élargissement en fuseau du canal mandibulaire gauche au niveau de l'angle mandibulaire. Panorex showing a large osteolytic unilocular lesion of the left mandible at the time of presentation following extraction of the first molar.

Figure 2 : Aspect histologique de la tumeur montrant un infiltrat principalement lymphocytaire, et des cellules fusiformes à petit noyaux, orientées (Antoni A) (a) ou désordonnées (b). (coloration MGG. X200)

Section ot the tumor displaying typical features: so-called Antoni A (a) and so-called Antoni B (b) tissues (Double stained MGG X200).

Figure 3 : Etude immuno-histo-chimique. La couleur brune révèle une forte positivité à la protéine $S-100$. (X400) Brown stains indicating that cytoplasm cells contains S-100 protein (X400). 
médecine buccale chirurgie buccale

)L. $8, \mathrm{~N}^{\circ} 1$

2001

page 40

\section{COMMENTAIRES}

Habituellement, les neurilemmomes sont des tumeurs isolées, à progression lente. La région de la tête et du cou est le plus souvent affectée. Différentes revues de la littérature confirment la faible fréquence de leur localisation orale et intra-osseuse. Kragh et coll. [9], dans une revue de 160 cas intéressant la tête et le cou, n'en dénombrent que 12 cas à localisation intraorale, dont 1 seul cas de localisation intra-osseuse intra-mandibulaire et 11 autres se situant au niveau des tissus mous (5 lingaux, 4 au plancher buccal et 2 jugaux). Das Gupta et coll. [10], sur 303 cas de tumeurs neurogènes solitaires bénignes, dénombrent 136 cas soit $45 \%$ de localisation cervico-céphalique, parmi lesquelles, seulement 16 cas sont de localisation intra-buccale.

Au niveau de la cavité buccale, les schwannomes peuvent se développer dans n'importe quel tissu mous. Hatziotis et coll. [11] dans une revue de la littérature de 1945 à 1964, réalisée sur 106 cas de tumeurs intra-orales concernant les tissus mous, dénombrent 59 cas au niveau de la langue, 11 au niveau du palais, 10 au niveau de la muqueuse, 10 au niveau du plancher, 7 au niveau des gencives, 6 au niveau des lèvres et 5 au niveau des vestibules. Cherrick et coll. [12], de leur côté, rapportent 18 cas de neurilemmomes intra-oraux dont aucun n'est intraosseux. En effet, les localisations intraosseuses sont rares et la mandibule est plus fréquemment touchée que le maxillaire. Sur 3987 cas de tumeurs osseuses primitives, Fawcett et coll. [13] dénombrent seulement 7 cas identifiés comme neurilemmomes intra-osseux dont 4 ont une localisation intra-mandibulaire. Shimura et coll. [14], dans une revue de 1938 à 1970, ne dénombrent que 10 cas de neurilemmomes intra-oraux dont 3 intra-osseux, 2 mandibulaires et 1 maxillaire faisant partie de la maladie de Recklinghausen.

Cette fréquence au niveau de la mandibule s'expliquerait entre autres, par le passage en son sein du nerf alvéolaire inférieur. Le site de prédilection de ces tumeurs mandibulaires se situe au niveau du ramus et du gonion [15]. Ide et coll. [16] dans leur revue de la littérature de
1937 à 1979 n'ont pu intégrer que 24 cas de neurilemmomes intra-osseux qu'ils ont jugés acceptables et un cas qu'ils ont rapporté. Depuis, Hietanen et coll. [17] en 1984 n'ont dénombré que 3 cas de neurilemmomes intraosseux dans la littérature et ont décrit deux nouveaux cas, tous mandibulaires.

La revue de la littérature entre 1984 et 1999 sur les schwannomes bénins intramandibulaires ne recense que 11 cas. Les revues de la littérature effectuées de 1937 à 1981 par Murphy et coll. [18] et de 1967 à 1985 par Artzi et coll. [19] (tableau 1) ne dénombrent que 40 cas de schwannomes bénins intra-mandibulaires.

Les schwannomes ne constituent que 1 à $9 \%$ des tumeurs buccales, ils peuvent survenir chez toutes les races et à n'importe quel âge. La moyenne d'âge est de 34 ans et $38 \%$ des malades sont âgés de 10 à 29 ans. Le sex-ratio femmes/homme est de $2 / 1$.

Cliniquement, les schwannomes centromandibulaires ont une progression tumorale lente et généralement asymptomatique.

Exceptionnellement, des signes neurologiques de type paresthésie peuvent être décrits.

Radiologiquement, ces tumeurs se présentent sous la forme d'une image radioclaire, homogène, uniloculaire bien délimitée par une paroi osseuse nette et possèdent un aspect similaire à d'autres lésions comme les kystes odontogéniques, l'angiome intraosseux, le granulôme eosinophile, ou même l'améloblastome. Dans le cas clinique rapporté, la lésion appendue à l'apex de la 36 , rappelle parfaitement une tumeur ostéolytique odontogène de type kyste péridentaire résiduel. Cet examen radiologique montre aussi et du même coté que la lésion un élargissement en fuseau du canal mandibulaire.

Macroscopiquement, lors de la tentative d'énucléation, la lésion ne possède pas de consistance granulo-kystique. Elle est plutôt d'aspect gélatineux entourant et ne refoulant pas le nerf alvéolaire inférieur. Comme pour la presque totalité des tumeurs ostéolytiques intra-mandibulaires le diagnostic final repose sur les résultats histopathologiques. 
Tableau 1 : Revue de littérature rapprochant celle effectuée de 1937 à 1981 par Murphy et coll. (a) et celle effectuée de 1967 à 1985 par Artzi et coll. (b). Cette revue est complétée par les auteurs depuis 1984 sur les localisations mandibulaires des schwannomes bénins ainsi que le cas rapporté (c).

\begin{tabular}{|c|c|c|}
\hline Auteurs & Année & Nombre et Site \\
\hline Zikens (a) & 1937 & 1 Mandibule \\
\hline Schroff (a) & 1945 & 1 Mandibule \\
\hline Baetz and Shacklford (a) & 1951 & 1 Mandibule \\
\hline Herrmann (a) & 1951 & 1 Mandibule \\
\hline Spilka (a) & 1953 & 1 Mandibule \\
\hline Aprile (a) & 1955 & 1 Mandibule \\
\hline Helsham (a) & 1956 & 1 Mandibule \\
\hline Ono (a) & 1958 & 1 Mandibule \\
\hline Bruce (a) & 1959 & 1 Mandibule \\
\hline Sveida (a) & 1959 & 1 Mandibule \\
\hline Sampter et al (a) & 1960 & 1 Mandibule \\
\hline Ackerman (a) & 1962 & 1 Mandibule \\
\hline Faraone (a) & 1963 & 1 Mandibule \\
\hline Fawcett and Dahlin (a) & 1967 & 4 Mandibule \\
\hline Morgan and Morgan (a) & 1968 & 1 Mandibule \\
\hline Hardt and Hardt (a) & 1972 & 1 Mandibule \\
\hline Shimura et coll. (a) (b) & 1973 & 1 Mandibule \\
\hline Sugimura et coll. (a) (b) & 1974 & 1 Mandibule (symphyse) \\
\hline Swangsilpa et coll. (a) (b) & 1976 & 1 Mandibule \\
\hline Ellis et coll. (a) (b) & 1977 & 2 Mandibule \\
\hline Rengaswamy (a) (b) & 1978 & 1 Mandibule \\
\hline Backer and Dunlap (a) (b) & 1979 & 1 Mandibule \\
\hline Scuibba and Sachs (b) & 1980 & 1 Mandibule \\
\hline Satterfield et coll. (a) (b) & 1981 & 1 Mandibule (symphyse) \\
\hline Schofield and Gardner (a) (b) & 1981 & 1 Mandibule \\
\hline Murphy and Giunta (a) (b) & 1984 & 1 Mandibule \\
\hline Hietanen et coll. (c) & 1984 & 2 Mandibule \\
\hline Marzola et coll. (c) & 1988 & 1 Mandibule (symphyse) \\
\hline Llewelyn et coll. (c) [21] & 1989 & 1 Mandibule \\
\hline Stevenson (c) [22] & 1989 & 1 Mandibule \\
\hline Musgrove and Moody (c) [23] & 1990 & 1 Mandibule \\
\hline Rubin et coll. (c) [24] & 1993 & 1 Mandibule \\
\hline Redman et coll. (c) [25] & 1996 & 1 Mandibule \\
\hline Belli et coll. (c)[ 26] & 1997 & 1 Mandibule \\
\hline Kimakhe et coll. (c) & 1999 & 1Mandibule \\
\hline
\end{tabular}

médecine buccale chirurgie buccale VOL. $8, \mathrm{~N}^{\circ}$ 2002 page 41
Histologiquement la tumeur entourée d'une capsule fibreuse, est constituée de deux populations cellulaires ; la première population cellulaires dite de type Antoni A est formée par des cellules fusiformes, regroupées en parallèle et en faisceaux, répandues dans un tissu conjonctif riche en collagène ; la microscopie électronique montre la présence d'une lame basale, la réaction histochimique à la protéine S-100 est positive. La deuxième population dite de type Antoni $B$, concerne des cellules moins nombreuses et moins bien ordonnées au sein d'un tissu conjonctif plus lâche; les cellules semblent être des cellules dégénérées de la population de type $A$; la microscopie électronique montre que ces cellules de type $B$ reposent sur une lame basale fragmentée et contiennent des lysosomes et des fragments de myéline. 
médecine buccale chirurgie buccale

)L. $8, N^{\circ} 1$ 2001 page 42
L'examen histopathologique et immuno-histochimique concluent en une tumeur nerveuse, un schwannome.

Les complications liées à ce type de lésion sont essentiellement neurologiques locales pouvant aller d'une simple paresthésie locale transitoire à une anesthésie totale et définitive du nef alvéolaire inférieur. En effet lors de la tentative d'énucléation, et vu la consistance de cette tumeur et son rapport avec le nerf alvéolaire inférieur, une exploration anatomique plus large est réalisée. Son objectif est d'obtenir une vision plus large du site opératoire afin d'orienter les gestes chirurgicaux avec la précision requise et de minimiser les risques de complication. De plus, une médication par voie générale d'anti-inflammatoires stéroïdiens à base de prednisolone à raison de $1 \mathrm{mg} / \mathrm{kg} / \mathrm{j}$ pendant 8 jours est prescrite à l'issue de l'intervention pour limiter l'inflammation locale. Malgré toutes les précautions, la patiente a manifesté en post-opératoire une légère paresthésie du territoire sensitif correspondant qui a cédé au bout de trois semaines.

Plusieurs cas rapportés ont montré la prévalence des deux types de neurofibromatose I et Il au niveau de la sphère cervico-faciale, sans pour autant faire la distinction entre eux. En général, la neurofibromatose est liée à des manifestations multiples, neurofibromes, schwannomes etc. Quatre situations peuvent se présenter, celle d'un schwannome isolé intramandibulaire, celle d'un schwannome multiple, celles d'une neurofibromatose de type I ou celle d'une neurofibromatose de type II.

Devant ces éléments du diagnostic histopathologique un examen neurologique orienté vers la recherche de lésions multiples s'intégrant ou non dans une maladie neurofibromateuse à été demandé. Cet examen a montré l'absence d'antécédent de neurofibromatose familiale héréditaire, l'absence de lésion cutanée de type «tâche café au lait» de Recklinghausen, l'absence de signe d'atteinte d'autres nerfs craniofaciaux autre que le nerf alvéolaire inférieur, en particulier le nerf acoustique. A cet examen neurologique a été associé des examens radiologiques : un scanner et une IRM. Le Scanner post-opératoire montre une normalité à gauche et à droite de l'entrée des canaux dentaires inférieurs (Fig. 4), alors que plus bas au niveau du corps mandibulaire, la tumeur est bien retrouvée sous la forme d'une tumeur refoulant harmonieusement les deux tables, sans éroder celles-ci (Fig. 5). Les examens en IRM n'ont montré aucune atteinte de type schwannome des branches du V, du VII et du VIII au niveau de la fosse postérieure.

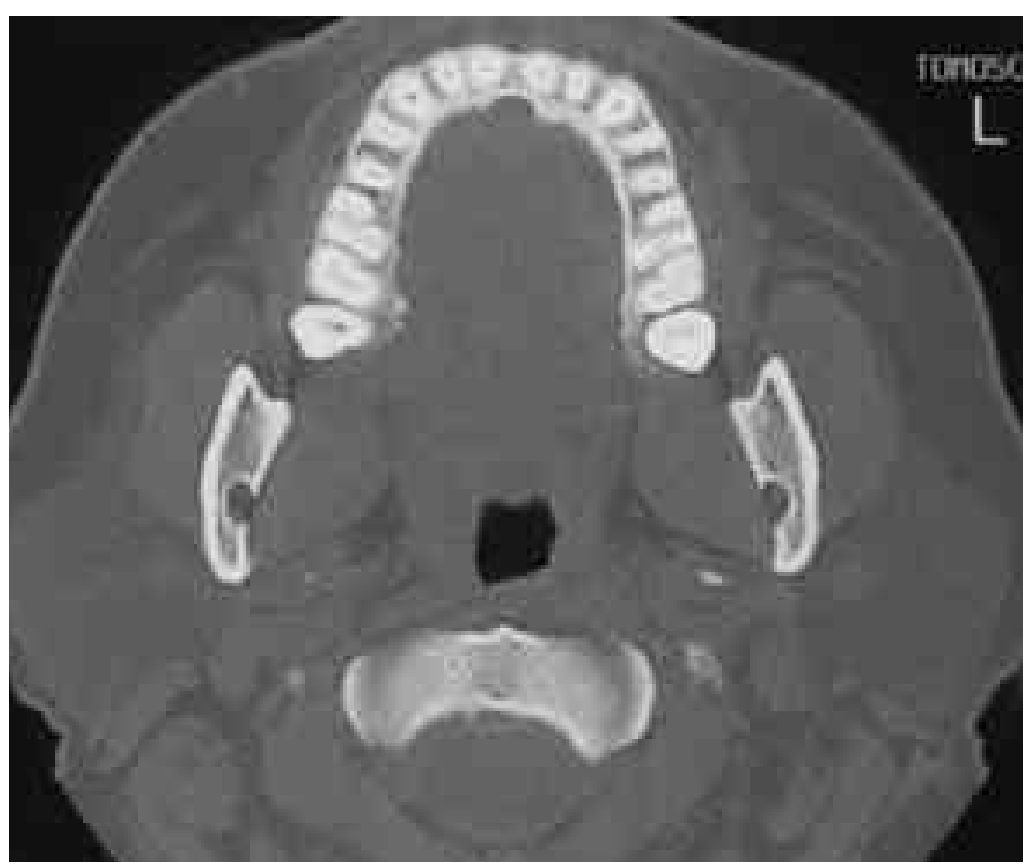

Figure 4 : Tomoscan en coupe coronale montre une normalité à gauche comme à droite de l'entrée des canaux des mandibulaires. CT-scan showing normal left and right lingulas. 
A travers la littérature, les cas rapportées de schwannome mandibulaires demeurent très rares. Dans le cas clinique présenté, cette tumeur bénigne neurogène, qui se présente appendue à l'apex de la 36, simule parfaitement un kyste périapical. Elle s'est avérée être un schwannome. Elle aurait, par ailleurs pu être inscrite dans une maladie neurologique. Les résultats des examens complémentaires ont permis de conclure à un schwannome isolé intra-mandibulaire.

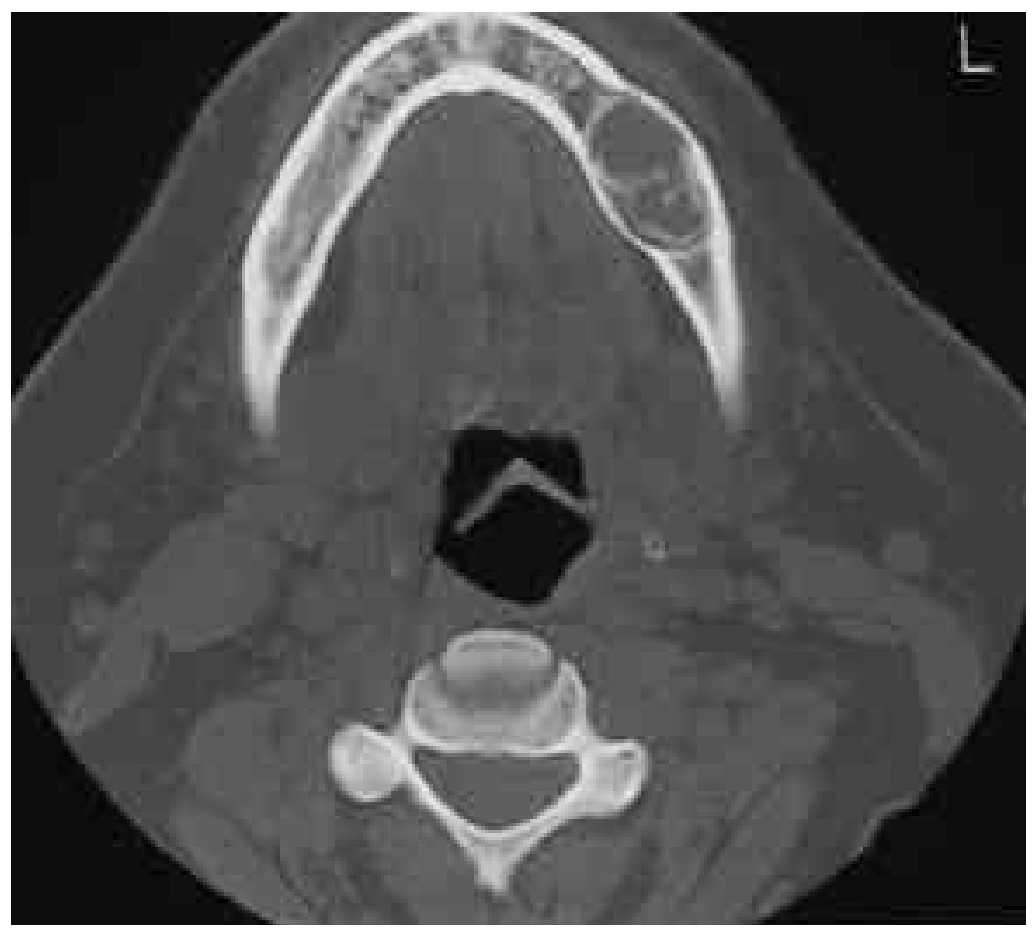

Figure 5 : Tomoscan en coupe coronale montre une image lacunaire centro-mandibulaire sans cloisonnement et refoulant harmonieusement les deux tables osseuses.

Coronal CT-scan showing a medial bony expansion.

\section{RÉFÉRENCES}

1 - Carstens PhB, S Chrodt GR. Malignant transformations of a benign encapsulated neurilemmoma. Am J Clin Pathol 1969; 51: 144-9.

2 - Redman RS, Guccion J G, Spector CJ, Keegan BP. Cellular schwannoma of the mandible: a case report with ultrastructural and immunohistochemical observations. J Oral-Maxillofac Surg 1996; 54: 339-44.

3 - Stout AP. The peripheral manifestations of the specific nerve sheath tumor. Am J Cancer 1935; 24: 751-96.

4 - Gorlin RJ, Goldman HM. Thomas oral pathology. (6th ed.) Volume 2, St. Louis, C.V Mosby Co., 1970.

5 - Sugimura M, Shirasuna, Yoshimura Y, Fuj imoto K, NAKAJ O Y. A case of neurilemmoma in the mandible. Int J Oral Surg 1974; 3: 194-200.

6 - WAGgener JD. Ultrastructure of benign peripheral nerve sheath tumors. Cancer 1966; 19: 699-709.
7 - J unqueira lCU, Montes GG, Kaupert D, Shigihara KM, BolonhaNI TM, KRISZTAN RM. Morphological and histochimical studies on the collagen in neurinomas, neurofibromas, and fibromas. I Neuropathol Exp Neurol 1981; 40: 123-33.

8 - Reychler H. Pathologie des maxillaires (pp 13901395). In : Traité de pathologies buccale et maxillofaciale. E. Piette, H. Reychler. éd : De Boeck Wesmael, Bruxelles, 1991.

9- Kragh V, S oule E, Masson J K. Benign and malignat neurilemmomas of the head and neck. Surg Gynecol Obstet 1960; 11: 211-8.

10 - Das Gupta TK, Brasfield RD, Strong EW, Hadjen SI. Benign solitary schwannomas (neurilemmomas), cancer 1969; 24: 355-65.

11 - HatZIOTIS M, Asprides H. Neurilemmoma (schwannoma) of the oral cavity. Oral Surg 1967; 24: 510-25. médecine buccale chirurgie buccale VOL. $8, \mathrm{~N}^{\circ}$ 2002 page 43 
12 - Cherrick HM, Eversol LR. Benign neural shealth neoplasm of the oral cavity. Report of 37 cases. Oral Surg Oral Med Oral Pathol 1971; 32: 900-9.

13 - FAWCETT KJ, DAHLIN DC. Neurilemmoma of bone. Am J Clin Pathol 1967; 47: 759-66.

14 - Shimura K, Allen EC, Kinoshita Y, Takaesu T. Central neurilemoma of the mandible: report of case and review of the literature. J Oral Surg 1973; 31: 363-7.

15 - Ord RA, Rennie J S. Central neurilemmoma of the maxilla. Report of a case and review of the literature. Int J Oral Surg 1981; 10: 137-9.

16 - Ide $F$, KanI Y, Hara K, Nakada M, UchiYama M, UmezaWa T, TOKokawa T. Central neurilemmoma of the literature. J Nipon Univ Sch Dent 1977; 19: 59-65.

17 - Hietanen J , M attila K, C alonius PE, AnkKuriniemı O, SuONPAa J, Happonen RP. Central neurilemmomas of the mandible. Report of a case. Int J Oral Surg 1984; 13: 166-71.

18 - MURPhY J , GiUnTA J L. Atypical central neurilemmoma of the mandible. Oral Surg Oral Med Oral Pathol 1985; 59: 275-8.

19 - ArtZI Z, TAICher S, NASS D. Neurilemmoma of the mental nerve. J Oral Maxillofac Surg 1991; 49: 200-4.
20-Marzola C, Borguetti MJ, Consolaro A. Neurilemmoma of the mandible. J Oral Maxillofac Surg 1988; 46: 330-4.

21 - Llewelyn J, Sugar AW. Neurilemmoma of the mandible. Report of a case. Br J Oral Maxillofac Surg 1989; 27: 512-6.

22 - SteVenson R. Central neurilemmoma (schwannoma) of the mandible. Case report. Aust Dent J 1989; 34: 410-3.

23 - Musgrove BT, Moody GH. Central neurilemma of the mandible. Br Dent J 1990; 169: 206-7.

24 - RUBIN MM, KoLL TJ . Central neurilemoma (schwannoma) of the mandible. NY State Dent J 1993; 59: 43-5.

25 - Redman RS, Guccion J G, Spector CJ, Keegan BP. Cellular schwannoma of the mandible: a case report with ultrastructural and immunohistochemical observations. J Oral Maxillofac Surg 1996; 54: 339-44.

26- Belli E, Becelli R, Matteini C, Lannetti G. Schwannoma of the mandible. J Craniofac Surg 1997; 8: 413-6. médecine buccale chirurgie buccale

L. $8, \mathrm{~N}^{\circ} 1$ 2001

page 44 